\title{
Nonlocal Effects Reflect the Jamming Criticality in Frictionless Granular Flows Down Inclines
}

\author{
Hugo Perrin, ${ }^{1,2}$ Matthieu Wyart, ${ }^{2}$ Bloen Metzger® ${ }^{1}$ and Yoël Forterre ${ }^{1}$ \\ ${ }^{1}$ Aix Marseille University, CNRS, IUSTI, 13453 Marseille, France \\ ${ }^{2}$ Institute of Physics, Ecole Polytechnique Fédérale de Lausanne, CH-1015 Lausanne, Switzerland
}

(Received 29 January 2021; revised 9 April 2021; accepted 6 May 2021; published 1 June 2021)

\begin{abstract}
The jamming transition is accompanied by a rich phenomenology such as hysteresis or nonlocal effects that is still not well understood. Here, we experimentally investigate a model frictionless granular layer flowing down an inclined plane as a way to disentangle generic collective effects from those arising from frictional interactions. We find that thin frictionless granular layers are devoid of hysteresis of the avalanche angle, yet the layer stability increases as it gets thinner. Steady rheological laws obtained for different layer thicknesses can be collapsed into a unique master curve, supporting the idea that nonlocal effects are the consequence of the usual finite-size effects associated with the presence of a critical point. This collapse indicates that the so-called isostatic length $l^{*}$, the scale on which pinning a boundary freezes all remaining floppy modes, governs the effect of boundaries on flow and rules out other propositions made in the past.
\end{abstract}

DOI: 10.1103/PhysRevLett.126.228002

Dense amorphous media close to the solid-liquid transition present a rich phenomenology such as hysteresis, finite-size, or nonlocal effects and dilatancy. Understanding these phenomena, which shape the jamming transition $[1,2]$, is a major challenge to describe the flow of granular media [3], foams [4], or soft glassy materials [5]. It is also important in geophysics to comprehend the occurence of earthquakes [6], landslides or avalanches [7,8]. The origin of these phenomena is still poorly understood and much debated. A striking example illustrating this situation is the well-known fact that the critical angle $\theta_{c}$ at which a granular layer flows under gravity depends on its thickness $h[9,10]$. The diversity of interpretations and models put forward to explain this property-successively attributed to dilatancy effects [11], to a diverging correlation length [12-15], to the consequence of nonlocal rheology [16-19], to boundary effects on mechanical stability [20], or to hysteresis [21] — highlights the difficulty in addressing the solid-liquid transition in amorphous media when many effects are potentially entangled.

Recently, we have developed a model granular system in which it is experimentally possible to tune, and even eliminate, solid friction between grains. This experimental system provides the means to highlight the frictional transition at the origin of shear thickening in dense particulate suspensions [22]. More importantly, when investigated in a rotating drum configuration, this model granular system revealed that both the Reynolds dilatancy effects and the hysteresis of the avalanche angle (the fact that the starting and stopping avalanche angles are different) disappear in the absence of interparticle friction $[22,23]$. Such a system of frictionless spheres thus provides a unique opportunity to study the flowing properties of granular layers without potential interplay with hysteretic behaviors or dilatancy effects.

About two decades ago, such an ideal granular material was studied numerically by Peynaud and Roux [24]. Since then, the investigation of frictionless particulate systems in numerical simulations, whether in the inertial or viscous regimes, has brought major contributions to the theoretical understanding of granular and suspension flows [25-28]. From a fundamental standpoint, this model granular material bridges frictional granular flows to other amorphous frictionless systems such as foams, emulsions, and glassy materials. It thus provides the appealing possibility of discriminating features that are specific to frictional interactions from the more generic ones that emanate from collective mechanical effects.

In this Letter, we investigate our model system of frictionless spheres in the inclined plane configuration, which enables us to both control the system size and impose a homogeneous friction coefficient $\mu$ on the medium. Our key findings are that (i) hysteresis of the avalanche angle is absent for frictionless grains whatever the thickness of the granular layer. By contrast, the layer stability and dynamics are strongly affected by finite-size effects. (ii) Flow rules obtained for different layer thicknesses collapse into a single master curve with a proper rescaling of variables. (iii) The latter result is explained by general finite-size scaling arguments near a critical point [29]. Our analysis suggests that the length scale on which flow is affected by a boundary is the so-called isostatic length $\ell^{*}$ associated with the jamming transition [30].

Experimental setup and protocols.-We use a model frictionless granular system composed of silica spheres of diameter $d=23.46 \pm 1.05 \mu \mathrm{m}$ [Fig. 1(a)]. When 
(a)
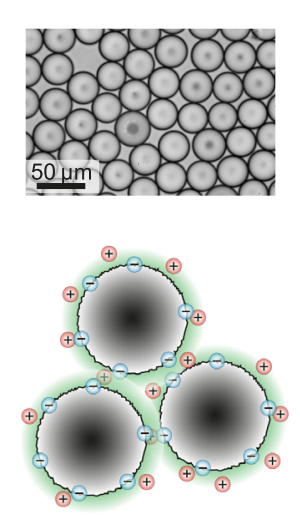

(b)

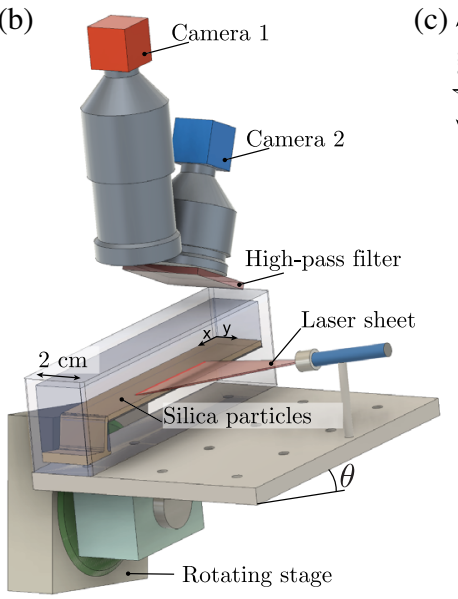

(c)

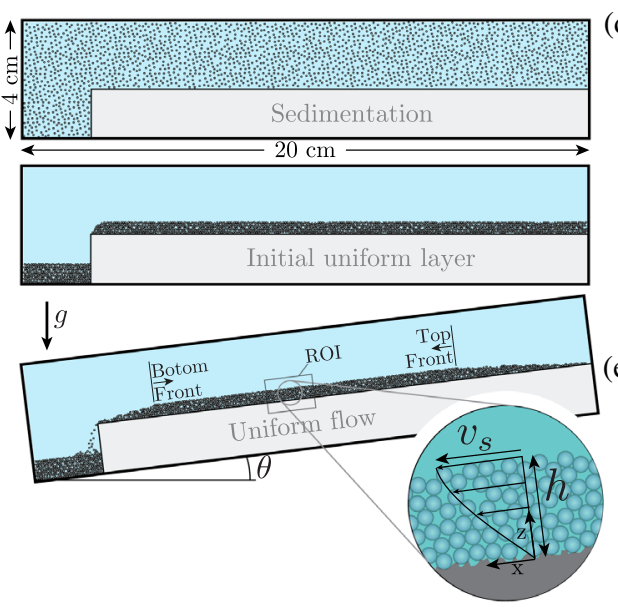

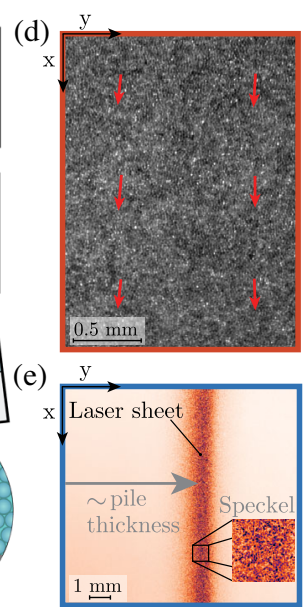

FIG. 1. (a) Top: Picture of the silica particles. Bottom: Schematic of the electrostatic repulsive forces preventing frictional particle contacts. (b) Schematic of the experimental setup. (c) Successive steps to prepare a uniform and presheared layer of grains. (d) Image from Camera 1 giving access to the particle surface velocity $v_{s}$ using particle image velocimetry. (e) Image from Camera 2 used to measure the pile thickness $h$ from the laser sheet position and the pile stability from the time correlation of the laser speckle.

immersed in pure water, the surface of these particles is negatively charged. Under the low external stress used in this study (below $10 \mathrm{~Pa}$ ), the resulting repulsive force [31] prevents the particles from making solid-frictional contacts. The particles then behave as frictionless spheres [22,23]. The inclined plane is the bottom of a parallelepiped container of size $20 \times 4 \times 2 \mathrm{~cm}^{3}$ [Fig. 1(b)]. It is covered with sandpaper (Grade P500 of roughness $\approx 30 \mu \mathrm{m}$ ) to insure rough boundary conditions. Both the inclined plane and the cameras are mounted on a high precision rotation stage (PI M-060), which controls the inclination angle $\theta$. To prepare the granular layer in a presheared configuration and at a uniform thickness $h$, we use the following protocol [see Fig. 1(c)]. The particles are first resuspended and then allowed to settle to form a layer of uniform thickness $h_{0} \approx 30 d>h$, with a typical flatness of $0.2 d$ per centimeter in the plane direction. The plane is then tilted at a large angle to initiate the flow and preshear the suspension. When the granular layer reaches the desired thickness $h$, measured using a laser sheet [Fig. 1(b)], the flow is stopped by setting the angle $\theta$ back to zero.

To determine the rheology of the flowing layer of grains, we use the fact that, in all our experiments, inertia is negligible (no acceleration terms) and the flow is uniform along the plane (no $x$ dependence). In this case, force balance implies that the suspension friction coefficient is homogeneous across the layer thickness and is given by the relation $\mu=\tau / P=\tan \theta$, where $\tau=\Delta \rho \phi g \sin \theta(h-z)$ is the shear stress, $P=\Delta \rho \phi g \cos \theta(h-z)$ is the normal stress, $\Delta \rho=850 \mathrm{~kg} / \mathrm{m}^{3}$ is the density difference between the particles and the suspending fluid, $\phi$ is the layer particle volume fraction, and $g$ is the gravity. Moreover, by imaging the layer surface, Camera 1 gives access to the grains' surface velocity $v_{s}$ using particle image velocimetry [Fig. 1(d)], and Camera 2 tracks the transverse position of the inclined laser sheet [Fig. 1(e)], thereby giving access to the layer thickness $h$, with an absolute resolution of $\pm 2.5 \mu \mathrm{m}$. These two measurements give access to the viscous number $J=\eta \dot{\gamma} / P=2 \eta v_{s} / h^{2} \phi \Delta \rho g \cos \theta$, where $\eta$ is the fluid viscosity and $\dot{\gamma}=2 v_{s} / h$ is the shear rate assuming a parabolic velocity profile [32] (see A in the Supplemental Material [33]). Here, the volume fraction is equal to the maximum packing of frictionless spheres, $\phi=0.64$, since measurements are performed in the limit of small viscous number $J<10^{-3}$. Note also that in all experiments, the stress from the fluid above the granular layer is negligible (see B in the Supplemental Material).

To characterize the stability of the flow and potential hysteretic behaviors of the avalanche angle, we developed a sensitive speckle correlation technique [34] able to detect particle velocities as small as $0.5 \mathrm{~nm} / \mathrm{s}$ (see $\mathrm{C}$ in the Supplemental Material).

Influence of the system size on hysteresis and layer stability.-We first address whether finite-size frictionless systems exhibit hysteretic behavior of the flow onset [see Fig. 2(a)]. Starting from a presheared granular layer having the desired thickness $h$, the inclination angle is increased to an initial angle $\theta_{0}\left[\theta_{0}=6.5^{\circ}\right.$ in Fig. 2(a)]. As shown in Fig. 2(a), the speckle correlation $C$ drops much below $C_{\text {th }}$ (red dashed line), indicating that the granular layer starts flowing. The angle of inclination $\theta$ is then successively decreased and increased using an interval halving procedure to determine and gradually refine the frontier between flow and arrest. For each step, the value of $\theta$ is maintained constant until the system relaxes to its steady state (flow or arrest), such that the protocol is independent of the rate of change of $\theta$. Note that, as the inclination angle always remains positive, the granular layer always flows down the plane in the same $x$ direction. Moreover, the flow rates involved here are sufficiently small 


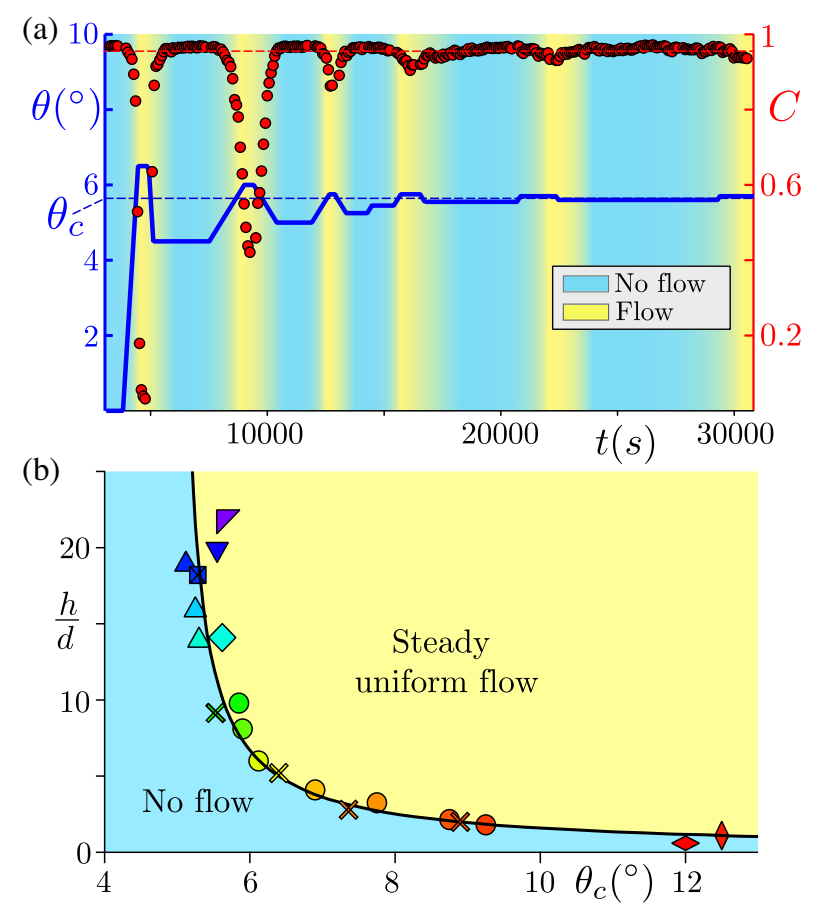

FIG. 2. Stability of a frictionless granular layer. (a) Interval halving procedure: controlled inclination angle $\theta$ (in blue) and resulting speckle correlation $C$ (in red) versus time for a layer of thickness $h / d \simeq 22$. Red dashed line: $C_{\mathrm{th}}=0.96$ delimiting flow $\left(C<C_{\text {th }}\right)$ from arrest $\left(C>C_{\text {th }}\right)$. Blue dashed line: critical stability angle $\theta_{c}(h / d)$. (b) Stability diagram: granular layer thickness $h / d$ versus critical stability angle $\theta_{c}$. Different open markers indicate different runs. Crosses correspond to the quasistatic friction coefficient $\mu_{c}(h / d)=\mu(J \rightarrow 0, h / d)$ obtained from Fig. 3(a). Black line: best fit with $h / d=$ $\left[\left(\tan \theta_{c}-\tan \theta_{c}^{\infty}\right) / a\right]^{-\alpha}$ yielding $\alpha=-0.9 \pm 0.1, a=0.15 \pm 0.03$, and $\theta_{c}^{\infty}=5.0^{\circ} \pm 0.1^{\circ}$.

$\left[v_{s} \approx \mathcal{O}\left(10^{-6}\right) \mathrm{m} / \mathrm{s}\right.$ corresponding to $\dot{h} \sim 0.2 d$ per hour) that during the whole protocol, the layer thickness remains constant within $0.5 d$, as directly checked. As illustrated in Fig. 2(a), the interval halving procedure converges to a unique critical inclination angle $\theta_{c}(h / d)$ determined with a resolution of $\pm 0.05^{\circ}$ (blue dashed line), and this occurs for all the layer thicknesses investigated $[1<h / d<22]$. We checked that the critical stability angle is independent of the choice of the initial angle $\theta_{0}$ of the interval halving procedure. These results show that a layer of frictionless particles stops or starts flowing at a unique critical angle $\theta_{c}(h / d)$ - the proof that no hysteresis of the avalanche angle is observed in frictionless granular systems, even of finite size.

The critical stability angle $\theta_{c}(h / d)$ is plotted in Fig. 2(b). For granular layers of thickness larger than $10 d$, the critical stability angle is nearly constant $\theta_{c}^{\infty} \simeq 5^{\circ}$ and in good agreement with previous experimental $\left(6^{\circ}\right.$ in [23]) and numerical results $\left(5.73^{\circ}\right.$ in [24]) obtained for frictionless systems in the infinite size limit. For thinner layers, however, the critical stability angle $\theta_{c}$ increases and, fitting the law

$$
h / d=\left[\left(\tan \theta_{c}-\tan \theta_{c}^{\infty}\right) / a\right]^{-\alpha},
$$

yields $\alpha=0.9 \pm 0.1, a=0.15 \pm 0.03$, and $\theta_{c}^{\infty}=5.0^{\circ} \pm$ $0.1^{\circ}$ (error bars set the parameter range for which residuals of the fit appear to behave randomly). These results show that, conversely to hysteresis, finite-size effects are still in play and significantly affect the stability of frictionless granular layers.

Influence of the system size on rheology.-We now study how finite-size effects influence the granular layer dynamic flowing properties in the aim of obtaining constitutive flow rules of the form $\mu(J, h / d)$. To this end, we fix the layer thickness $h$ and take advantage of the setup to measure $J$ while imposing a quasistatic decreasing ramp of $\mu=\tan \theta$ using the rotation stage. This quasisteady protocol provides, in a single measurement, the full rheological laws $\mu(J, h / d)$ for a given value of $h / d$. We checked that true steady state measurements (constant $\mu$ ) give the same results (see D in the Supplemental Material).

The rheological laws $\mu(J, h / d)$ are shown in Fig. 3(a) for various layer thickness $h / d$ and for a wide range of $J$. We recover that the system size has a strong effect on the flow threshold. The quasistatic friction coefficient $\mu_{c}(h / d)=\mu(J \rightarrow 0, h / d)$ (crosses) obtained from dynamically ramping down $\mu$ are fully consistent with the critical stability angle obtained from the "static" interval halving procedure procedure shown in Fig. 2(b), confirming the robustness of our finite-size effect characterization. Moreover, plotting the difference $\mu(J, h / d)-\mu_{c}(h / d)$ as function of $J$ shows that the system size also affects the flow dynamical properties [Fig. 3(a), inset]. The large system $(h / d \simeq 18.2)$ exhibits a power law with an exponent of 0.38 , in close agreement to the exponents measured experimentally in a rotating drum $(0.37 \pm 0.05$ [23]) and derived theoretically $(\beta=0.35$ [28], from [35,36]) for frictionless spheres in the infinite size limit. However, the power law exponent systematically increases as the system size is decreased.

Together with Fig. 2(b) and Eq. (1), these observations support the two following asymptotic regimes:

$$
\begin{gathered}
\mu(J \rightarrow 0, h / d) \sim \mu_{c}^{\infty}+a(h / d)^{-1 / \alpha}, \quad \text { and } \\
\mu(J, h / d \rightarrow \infty) \sim \mu_{c}^{\infty}+b J^{\beta} .
\end{gathered}
$$

To rationalize the rheological laws $\mu(J, h / d)$, we use the framework of phase transitions and the usual finite-size scaling assumption valid near a critical point [29], which was recently used to characterize other aspects of the jamming transition, including the fluctuations of the critical packing fraction $\phi_{c}$ [37] or the characteristic strain needed to jam a packing [38,39]. In this view, $J$ is the order parameter controlled by the excess macroscopic friction $\Delta \mu \equiv \mu-\mu_{c}^{\infty}$ and system size $h$ : 


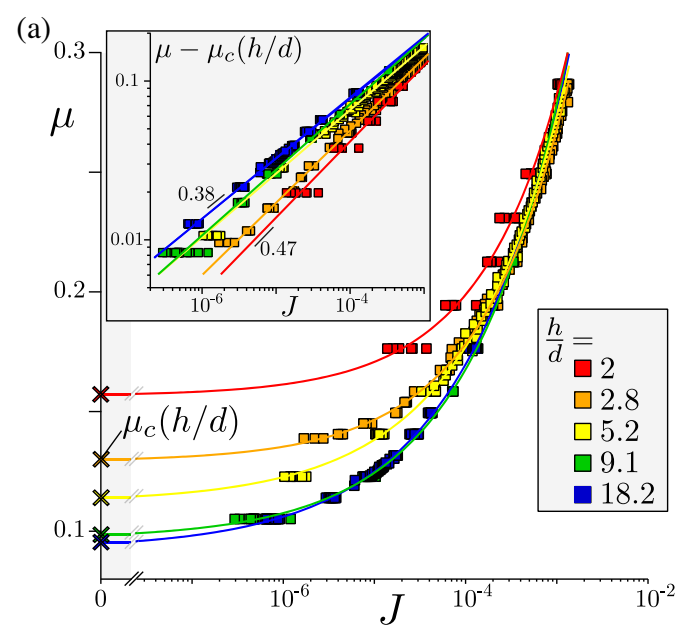

(b)

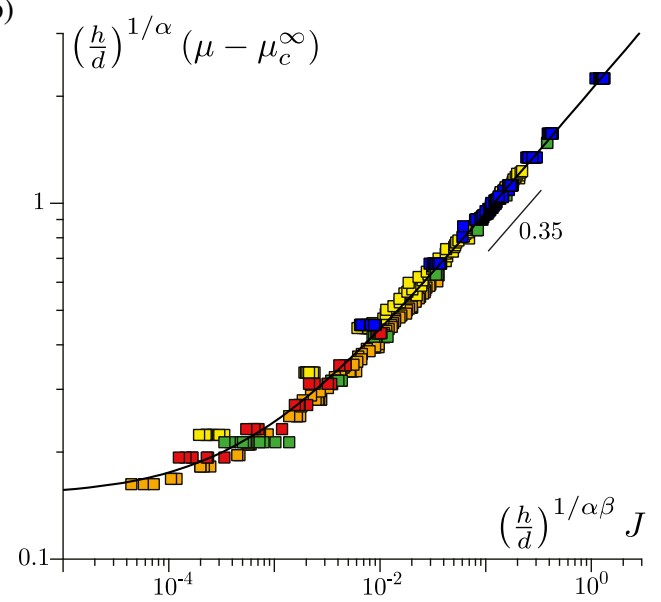

FIG. 3. (a) Macroscopic friction coefficient $\mu$ versus viscous number $J$ for different layer thicknesses $h / d$. Solid lines: Best fits with $\mu=\mu_{c}+b^{\prime} J^{\beta^{\prime}}$. The values of $\mu_{c}(h / d)$ (crosses) are reported in Fig. 2(b) using the relation $\mu_{c}(h / d)=\tan \theta_{c}(h / d)$. Inset: Same data plotting the reduced macroscopic friction $\mu-\mu_{c}(h / d)$ versus $J$. (b) Rescaled flow rule plotting $(h / d)^{1 / \alpha}\left(\mu-\mu_{c}^{\infty}\right)$ versus $(h / d)^{1 / \alpha \beta} J$ with $\alpha=0.9$ and $\beta=0.35$. Black line: $g(x)=a+\left(c x^{\gamma}\right)\left(b x^{\beta}\right) /\left(b x^{\beta}+c x^{\gamma}\right)$, with $a=0.15, b=2.0 \pm 0.1$, $c=29 \pm 5$, and $\gamma=0.73 \pm 0.05$.

$$
J(\Delta \mu, h)=\Delta \mu^{1 / \beta} f[h / \xi(\Delta \mu)],
$$

where $f$ is some scaling function and $\xi(\Delta \mu)$ is a diverging length scale. This scaling form is consistent with Eq. (2) if $\xi(\Delta \mu)=h\left(\theta_{c}\right) / c_{0} \sim d \Delta \mu^{-\alpha}$, where $c_{0}$ is some constant and $f\left(c_{0}\right)=0$, while Eq. (3) imposes $f(\infty)=b^{-1 / \beta}$. Equation (4) can be rewritten as

$$
\Delta \mu(J, h)=(h / d)^{-1 / \alpha} g\left[(h / d)^{1 / \alpha \beta} J\right],
$$

where $g$ is some function. This result implies that the data of Fig. 3(a) should collapse on a single curve by plotting the rescaled friction coefficient $(h / d)^{1 / \alpha} \Delta \mu(J, h)$ as a function of the rescaled viscous number $(h / d)^{1 / \alpha \beta} J$. Remarkably, the collapse shown in Fig. 3(b) is excellent without any fitting parameters [since $\beta$ is theoretically predicted and $\alpha$ extracted from Fig. 2(b)]. This description unifies both flow properties and arrest. Quantitatively, we find that the scaling function $g$ is well-approximated by $g(x)=$ $a+b c x^{\gamma+\beta} /\left(b x^{\beta}+c x^{\gamma}\right)$ with $a=0.15, \quad b=2.0 \pm 0.1$, $c=29 \pm 5$, and $\gamma=0.73 \pm 0.05$ [black line in Fig. 3(b)].

Diverging length scale.-We now propose a scaling argument for the exponent $\alpha \approx 0.9$ characterizing the observed finite-size effects in frictionless granular flows. We use two facts: (i) in packing of particles, there exists a diverging "isostatic length scale" $\ell^{*} \sim d /|\delta z|$ (where $\delta z=z-z_{c}^{\infty}$ and $z_{c}^{\infty}$ is the threshold "isostatic" coordination where the infinite system rigidifies [40]) that characterizes how pinning a boundary affects linear properties of the material. Specifically, $\ell^{*}$ controls both the elasticity of overconstrained materials [30] and the scale on which pinning boundaries rigidify a floppy, underconstrained system [41]. We posit that $\ell^{*}$ is also the length scale on which a granular flow is affected by the presence of a boundary, i.e., $h\left(\theta_{c}\right) \sim \ell^{*} \sim d /|\delta z|$. (ii) In flowing systems of hard frictionless particles, increasing the macroscopic friction breaks more contact and reduces the coordination. The microscopic theory of [28] predicts $|\delta z| \sim \Delta \mu^{0.83}$ both for overdamped and inertial flows, in good agreement with numerics. Putting these two results together, we find that jamming occurs when the added constraints from the boundary balance the degrees of freedom of the bulk, leading to $h\left(\theta_{c}\right) \sim d \Delta \mu^{-\alpha}$ with $\alpha=0.83$, in close agreement with our observation $\alpha=0.9 \pm 0.1$. Note that another popular length scale that diverges near jamming, $\ell_{c} \sim d / \sqrt{|\delta z|}$ [42], which characterizes the response to a point perturbation [41], is ruled out as it would lead to $\alpha=0.41$ incompatible with our data.

Discussion.-In this Letter, we have shown experimentally that a finite-size frictionless granular layer is devoid of hysteresis: a granular layer of thickness $h / d$ stops or starts flowing at unique critical stability angle $\theta_{c}(h / d)$. Nonetheless, thinner layers are more stable, indicating that finite-size effects are still in play even without solid friction, as previously observed with foams [4]. These findings highlight the fact that no hysteretic behaviors emerge from finite-size effects and that hysteresis is a feature entirely rooted to the presence of interparticle friction $[23,28]$.

The critical stability angle is found to follow $h / d \sim\left(\tan \theta_{c}-\tan \theta_{c}^{\infty}\right)^{-\alpha}$ with $\alpha \approx 0.9 \pm 0.1$ [Eq. (1) and Fig. 2(b)]. Our results clearly reject mechanisms based on dilatancy effects as an explanation for this behavior as frictionless granular layers are not dilatant [24]. The unicity of the critical curve separating flow and arrest $\left(h_{\text {start }}=h_{\text {stop }}\right)$ also challenges mechanisms based on flowinduced mechanical noise [16,17] or granular fluidity [19], since dynamic noise is absent when starting from a static configuration. Our experimental work thus called for a new coherent explanation. Here we have argued that nonlocal 
effects are a necessary consequence of the finite-size effects near a critical point, justifying the remarkable collapse of flow curves for different thicknesses into a single master curve. The extracted diverging length scale is consistent with the isostatic length scale following $\ell^{*} \sim d \Delta \mu^{-0.83}$, which is predicted to hold also for inertial frictionless systems [28].

Here, we have generalized a boundary dependent argument from packings (the effect of $\ell^{*}$ ) to describe the influence of a wall on a thin granular layer. Such a generalization is not straightforward. Future works should investigate whether this argument can also be applied to other types of boundaries, such as plane shear or heap flow confined between walls. Another key question is its generalization to the frictional case. In [43], it was argued that the role of excess coordination in frictionless particles is then replaced by the fraction of sliding contact for frictional ones. The latter appears to be proportional to $\Delta \mu$. Following the above length-scale argument, we would obtain $\alpha=1$, which is consistent with observations $[9,20]$.

We thank O. Pouliquen and S. Mandal for discussions and S. Noël for building the incline. Support: European Research Council under the European Union Horizon 2020 Research and Innovation program (ERC Grant Agreement No. 647384), ANR ScienceFriction (ANR-18-CE300024), by the Swiss National Science Foundation for support under Grant No. 200021-165509 and the Simons Foundation Grant No. 454953 (M. W.).

[1] C. S. O’Hern, L. E. Silbert, A. J. Liu, and S. R. Nagel, Phys. Rev. E 68, 011306 (2003).

[2] P. Olsson and S. Teitel, Phys. Rev. Lett. 99, 178001 (2007).

[3] Y. Forterre and O. Pouliquen, Annu. Rev. Fluid Mech. 40, 1 (2008).

[4] R. Lespiat, S. Cohen-Addad, and R. Höhler, Phys. Rev. Lett. 106, 148302 (2011).

[5] J. Goyon, A. Colin, and L. Bocquet, Soft Matter 6, 2668 (2010).

[6] J. Leeman, D. Saffer, M. Scuderi, and C. Marone, Nat. Commun. 7, 11104 (2016).

[7] A. Lucas, A. Mangeney, and J. P. Ampuero, Nat. Commun. 5, 3417 (2014).

[8] B. Ferdowsi, C. P. Ortiz, and D. J. Jerolmack, Proc. Natl. Acad. Sci. U.S.A. 115, 4827 (2018).

[9] O. Pouliquen, Phys. Fluids 11, 542 (1999).

[10] A. Daerr and S. Douady, Nature (London) 399, 241 (1999).

[11] O. Pouliquen and N. Renaut, J. Phys. II (France) 6, 923 (1996).

[12] D. Ertaş and T. C. Halsey, Europhys. Lett. 60, 931 (2002).

[13] O. Pouliquen, Phys. Rev. Lett. 93, 248001 (2004).

[14] P. Mills, P. Rognon, and F. Chevoir, Europhys. Lett. 81, 64005 (2008).
[15] T. Gueudré, J. Lin, A. Rosso, and M. Wyart, Soft Matter 13, 3794 (2017).

[16] O. Pouliquen, Y. Forterre, and S. Le Dizes, Adv. Complex Syst. 04, 441 (2001).

[17] A. Lemaitre, Phys. Rev. Lett. 89, 064303 (2002).

[18] I. S. Aranson and L. S. Tsimring, Phys. Rev. E 65, 061303 (2002).

[19] K. Kamrin and D. L. Henann, Soft Matter 11, 179 (2015).

[20] M. Wyart, Europhys. Lett. 85, 24003 (2009).

[21] A. Edwards, A. Russell, C. Johnson, and J. Gray, J. Fluid Mech. 875, 1058 (2019).

[22] C. Clavaud, A. Bérut, B. Metzger, and Y. Forterre, Proc. Natl. Acad. Sci. U.S.A. 114, 5147 (2017).

[23] H. Perrin, C. Clavaud, M. Wyart, B. Metzger, and Y. Forterre, Phys. Rev. X 9, 031027 (2019).

[24] P.-E. Peyneau and J.-N. Roux, Phys. Rev. E 78, 011307 (2008).

[25] G. Combe and J.-N. Roux, Phys. Rev. Lett. 85, 3628 (2000).

[26] S. Gallier, E. Lemaire, F. Peters, and L. Lobry, J. Fluid Mech. 757, 514 (2014).

[27] R. Mari, R. Seto, J. F. Morris, and M. M. Denn, J. Rheol. 58, 1693 (2014).

[28] E. DeGiuli, G. Düring, E. Lerner, and M. Wyart, Phys. Rev. E 91, 062206 (2015).

[29] J. Cardy, Finite-Size Scaling (Elsevier, New York, 2012).

[30] M. Wyart, S. R. Nagel, and T. A. Witten, Europhys. Lett. 72, 486 (2005).

[31] J. N. Israelachvili, Intermolecular and Surface Forces (Academic Press, New York, 2011).

[32] C. Cassar, M. Nicolas, and O. Pouliquen, Phys. Fluids 17, 103301 (2005).

[33] See Supplemental Material at http://link.aps.org/ supplemental/10.1103/PhysRevLett.126.228002 for additional experimental information.

[34] M. Erpelding, A. Amon, and J. Crassous, Phys. Rev. E 78, 046104 (2008).

[35] P. Charbonneau, J. Kurchan, G. Parisi, P. Urbani, and F. Zamponi, J. Stat. Mech. 10 (2014) P10009.

[36] P. Charbonneau, J. Kurchan, G. Parisi, P. Urbani, and F. Zamponi, Nat. Commun. 5, 3725 (2014).

[37] D. Vågberg, D. Valdez-Balderas, M. Moore, P. Olsson, and S. Teitel, Phys. Rev. E 83, 030303(R) (2011).

[38] A. H. Clark, J. D. Thompson, M. D. Shattuck, N. T. Ouellette, and C. S. O’Hern, Phys. Rev. E 97, 062901 (2018).

[39] J. D. Thompson and A. H. Clark, Phys. Rev. Research 1, 012002(R) (2019).

[40] J. C. Maxwell, London, Edinburgh, Dublin Philos. Mag. J. Sci. 27, 294 (1864).

[41] G. Düring, E. Lerner, and M. Wyart, Phys. Rev. E 89, 022305 (2014).

[42] L. E. Silbert, A. J. Liu, and S. R. Nagel, Phys. Rev. Lett. 95, 098301 (2005).

[43] E. DeGiuli and M. Wyart, Proc. Natl. Acad. Sci. U.S.A. 114, 9284 (2017). 